\title{
ASPECTOS TÉCNICOS DA MONITORIZAÇÃO DA PRESSÃO INTRACRANIANA PELO MÉTODO SUBARACNÓIDEO NO TRAUM ATISMO CRANIENCEFÁLICO GRAVE
}

\author{
Venâncio Pereira Dantas Filho" ${ }^{1}$, Antonio Luis Eiras Falcão², Luis Antonio da Costa Sardinha², \\ José Jorge Facure², Sebastião Araújo², Renato G.G. Terzi²
}

\begin{abstract}
RESUMO - Foram analisados prospectivamente 206 pacientes com traumatismo craniencefálico (TCE) grave (8 pontos ou menos na Escala de Coma de Glasgow), internados na Unidade de Terapia Intensiva do Hospital das Clínicas da Universidade Estadual de Campinas. Após avaliação por tomografia computadorizada de crânio (TC), 72 pacientes necessitaram de tratamento neurocirúrgico. Todos os pacientes foram submetidos à monitorização contínua da pressão intracraniana (PIC) pelo método subaracnóideo (11 com parafuso metálico e 195 com cateter plástico). Os níveis de PIC foram registrados continuamente na tela do monitor, sendo os seus valores de final de hora anotados em ficha padronizada. Todos os pacientes foram tratados segundo um protocolo orientado pelos níveis da PIC. Não foram observadas complicações hemorrágicas ou hematomas intracranianos relacionados ao método de monitorização em TC de controle. Para controle de infecções intracranianas, foram colhidas amostras de líquido cefalorraquidiano através de punção lateral C1-C2 em 66 pacientes com PIC abaixo de $20 \mathrm{~mm} \mathrm{Hg}$, sendo positivas as culturas para Acinetobacter sp em 2 pacientes. 0 resultado final na alta hospitalar mostrou $75(36,40 \%)$ óbitos e $131(63,6 \%)$ sobreviventes. Os níveis de PIC influenciaram significativamente o resultado final $(p<0,001)$, o que confirma a importância de sua monitorização e controle no tratamento do TCE grave. O método subaracnóideo para a monitorização contínua da PIC foi considerado aplicável, seguro, simples, de baixo custo e útil para a orientação do tratamento. A metodologia de registro da PIC foi considerada útil e prática. Apesar dos avanços técnicos nesta área, o método subaracnóideo mostrou-se uma alternativa bastante viável para a monitorização da PIC em pacientes com traumatismo craniencefálico grave.
\end{abstract}

PALAVRAS-CHAVE: traumatismo craniencefálico, pressão intracraniana, escala de coma de Glasgow, coma.

\section{Technical aspects of intracranial pressure monitoring by subarachnoid method in severe head injury}

ABSTRACT - Two hundred and six patients with severe head injury (Glasgow Coma Scale of 8 points or less after nonsurgical resuscitation on admission), managed at Intensive Care Unit-Hospital das Clínicas - Universidade Estadual de Campinas were prospectively analysed. All patients were assessed by CT scan and 72 required neurosurgical intervention. All patients were continuously monitored to evaluate intracranial pressure (ICP) levels by a subarachnoid device (11 with subarachnoid metallic bolts and 195 with subarachnoid polyvinyl catheters). The ICP levels were continuously observed in the bedside pressure monitor display and their endhour values were recorded in a standard chart. The patients were managed according to a standard protocol guided by the ICP levels. There were no intracranial haemorrhagic complications or hematomas due the monitoring method. Sixty six patients were punctured by lateral C1-C2 technique to assess infectious complications and 2 had positive cerebrospinal fluid samples for Acinetobacter sp. The final results measured at hospital discharge showed 75 deaths $(36,40 \%)$ and $131(63,60 \%)$ survivors. ICP levels had significantly influenced the final results $(p<0,001)$. The subarachnoid method to continuously assess the ICP levels was considered aplicable, safe, simple, low cost and useful to advise the management of the patients. The ICP record methodology was practical and useful. Despite the current technical advances the subarachnoid method was considered viable to assess the ICP levels in severe head injury.

KEY WORDS: head injury, intracranial pressure, Glasgow coma scale, coma.

'Disciplina de Neurocirurgia do Departamento de Neurologia e Neurocirurgia da Faculdade de Ciências Médicas da Universidade Estadual de Campinas (UNICAMP) - Campinas SP, Brasil; ${ }^{2}$ Unidade de Terapia Intensiva do Hospital das Clínicas da UNICAMP

Recebido 21 Fevereiro 2001, recebido na forma final 8 Agosto 2001. Aceito 20 Agosto 2001.

Dr. Venâncio Pereira Dantas Filho - Av Estados Unidos, 219 - 13036-390 Campinas SP - Brasil. E-mail: venancio@hc.unicamp.br 
Muitos esforços têm sido dispendidos para minimizar o impacto social do traumatismo craniencefálico (TCE), visando melhorar a prevenção, o atendimento pré-hospitalar, o tratamento intra-hospitalar e a reabilitação desses pacientes. A hipertensão intracraniana (HIC) pós-traumática aguda é complicação grave e com forte influência negativa sobre a evolução de grande número de pacientes com TCE, principalmente naqueles com maior gravidade. Métodos de quantificação dos níveis da presão intracraniana (PIC) e de controle da HIC têm sido propostos, mas a sua implementação ainda permanece um desafio para grande parte dos hospitais brasileiros e de outros países em desenvolvimento. Custos, praticabilidade, confiabilidade e riscos de complicações são aspectos importantes, muitas vezes limitantes, para a escolha do método de monitorização da PIC.

Relatamos nossa experiência com a utilização do método subaracnóideo com parafuso metálico e cateter plástico na Unidade de Terapia Intensiva (UTI) de adultos do Hospital das Clínicas da Universidade Estadual de Campinas (HC-UNICAMP), que possibilitou a implantação de um protocolo de tratamento do TCE grave orientado pela medida contínua dos níveis da PIC, racionalizando o uso de medidas terapêuticas específicas para a HIC.

\section{MÉTODO}

Foram selecionados prospectivamente 206 pacientes consecutivos com TCE grave, ou seja, com 8 pontos ou menos na Escala de Coma de Glasgow (ECGI) ${ }^{1}$ pós-reanimação, internados e tratados na fase aguda na UTI de adultos do HC-UNICAMP, no período de junho de 1989 a janeiro de 1995. Desses pacientes, 166 eram do sexo masculino $(80,58 \%)$ e 40 do feminino $(19,42 \%)$. A média de idade foi 29,21 anos, com mediana de 25 anos, variando de 11 a 74 anos. As causas do TCE foram acidentes de trânsito (automóvel, motocicleta e atropelamentos) em 147 casos $(71,36 \%)$, quedas em 37 casos $(17,96 \%)$ e os traumas diretos sobre o segmento cefálico por queda de objetos ou agressões em 15 casos $(7,28)$. A causa do TCE não foi conhecida em 7 pacientes $(3,40 \%)$. Apresentaram pontuação entre 3 e 5 pontos na ECGI na avaliação inicial 90 pacientes $(43,69 \%)$, enquanto $116(56,31 \%)$ apresentaram pontuação entre 6 e 8 pontos.

Foram excluídos os pacientes vítimas de ferimentos cranianos por projétil de arma de fogo. Para minimizar os efeitos de tamponamento sobre a monitorização da PIC, foram excluídos pacientes com falhas ósseas cranianas maiores que $5 \mathrm{~cm}$ de diâmetro, bem como aqueles nos quais a dura-máter foi deixada aberta nos procedimentos cirúrgicos necessários. A identificação de fístulas liquóricas nos pacientes não foi considerada critério de exclusão nesta casuística.

Após os procedimentos de reanimação (principalmen- te intubação traqueal e reposição volêmica) no atendimento inicial no pronto-socorro (PS), todos os pacientes foram submetidos à tomografia computadorizada de crânio (TC) e 72 pacientes (34,95\%) necessitaram de tratamento neurocirúrgico, sendo em seguida encaminhados para internação na UTI. Nestes pacientes o dispositivo subaracnóideo para a monitorização da PIC foi implantado no centro cirúrgico, imediatamente após o procedimento neurocirúrgico necessário. Os pacientes sem indicação de tratamento neurocirúrgico foram encaminhados para internação na UTI o mais rapidamente possível, conforme disponibilidade de vagas da unidade.

Uma vez internados na UTI, tendo sido corrigidas todas as alterações sistêmicas possíveis, os pacientes foram mantidos em tratamento geral para o TCE grave, que compreendeu as seguintes medidas ${ }^{2-4}$ : 1- Decúbito elevado $30^{\circ}$. 2- Sedação com thionembutal (dose entre 0,5 a 2,0 mg/ $\mathrm{kg} / \mathrm{h}$ ) ou com fentanil associado a midazolan em pacientes com instabilidade cardiocirculatória $(0,5 \mathrm{mg}$ de fentanil e $75 \mathrm{mg}$ de midazolan em $250 \mathrm{ml}$ de soro glicosado a $5 \%$, com gotejamento inicial de 10 a 20 microgotas/min, ou em doses suficientes para sedação, de acordo com a resposta individual do paciente). 3- Ventilação mêcanica com os parâmetros do ventilador ajustados para $\mathrm{PaCO}_{2}$ entre 30 e $35 \mathrm{~mm} \mathrm{Hg}$.

Os pacientes ainda não monitorizados foram submetidos à implantação de dispositivo subaracnóideo para a monitorização da PIC, à beira do leito e com técnica asséptica (gorro, máscara, aventais e campos estéreis). Esse procedimento seguiu a seguinte padronização técnica ${ }^{2-4}$ : 1Infiltração anestésica convencional, após tricotomia de todo o couro cabeludo, assepsia da região pré-coronal direita e colocação de campos estéreis. 2- Incisão coronal linear do couro cabeludo de $3 \mathrm{~cm}$ de comprimento, a 3 $\mathrm{cm}$ de distância da linha sagital mediana e retração dos bordos da incisão. 3- Trepanação manual da calota craniana (broca tipo Cushing de $1 / 2$ polegada). 4a- Abertura cruciforme ampla da dura-máter com bisturi lâmina $n^{0}$ 11 até saída de líquido cefalorraquidiano (LCR) e colocação, por rosqueamento ao osso, de parafuso metálico tipo Richmond modificado ${ }^{5}$, com chave apropriada (conjunto de pressão intracraniana: plessímetro com câmara para fixação e chave cruzada - IMED - Instrumental Médico do Brasil Ltda., Rio de Janeiro, R.J., Brasil). 4b- Abertura linear sagital da dura-máter, com bisturi lâmina $\mathrm{n}^{0} 11$ até saída de LCR e introdução subaracnóidea de $3 \mathrm{~cm}$ de cateter de aspiração traqueal $n^{0} 8$ (sonda de aspiração traqueal - Ibras CBO - Indústrias Cirúrgicas e Ópticas S.A., Campinas, S.P., Brasil), com saída por contra-abertura em túnel subcutâneo de $5 \mathrm{~cm}$, posterior à incisão. 5 - Fechamento do couro cabeludo em um único plano com pontos separados em redor do parafuso ou, no caso do cateter, fechamento de toda a incisão em plano único com pontos separados, com a fixação do cateter ao couro cabeludo em sua saída pela contra-abertura com ponto único. 6- Preenchimento de todo o sistema com soro fisiológico e conexão do parafuso ou do cateter ao tubo de polietileno 
do domo do sistema de registro de pressão (Monitor Multiprogramável SDM 2000 - DIXTAL Tecnologia Ind. Com. Ltda., Santo Amaro, S.P., Brasil). 7- Curativo oclusivo das incisões.

O sistema foi calibrado tendo como nível zero a pressão atmosférica, na altura do conduto auditivo externo do paciente.

Foram monitorizados 11 pacientes $(5,34 \%)$ com parafuso e 195 pacientes $(94,66 \%)$ com cateter. O tempo médio de monitorização foi de 5,22 dias, com uma mediana de 5 dias, mínimo de 1 e máximo de 14 dias.

A curva e os valores da PIC foram registrados continuamente na tela do monitor, sendo o seu valor de final de hora anotado pelo pessoal de enfermagem em ficha padronizada da UTI. Foram considerados normais os níveis de PIC até $10 \mathrm{~mm} \mathrm{Hg}$, níveis até $20 \mathrm{~mm} \mathrm{Hg}$ foram considerados aceitáveis (HIC leve), não necessitando de tratamento específico. Níveis de PIC acima de $20 \mathrm{~mm} \mathrm{Hg}$ foram considerados HIC moderada, sendo instituído tratamento específico. Níveis de PIC acima de $40 \mathrm{~mm} \mathrm{Hg}$ foram considerados HIC grave.

Nos pacientes que mantiveram valores da PIC iguais ou abaixo de $20 \mathrm{~mm} \mathrm{Hg}$ foram mantidas as medidas terapêuticas gerais. Uma vez mantidos esses níveis pelo menos por $48 \mathrm{hs}$, foram iniciados procedimentos de retirada da sedação, ventilação mecânica e monitorização da PIC. Nos pacientes em que a PIC se manteve acima de $20 \mathrm{~mm}$ $\mathrm{Hg}$, foi iniciado tratamento específico, progressivo e cumulativo para a $\mathrm{HIC}^{2-4}$. Cada nova conduta só foi iniciada após a verificação da refratariedade da PIC à conduta anterior: 1- Hiperventilação com níveis de $\mathrm{PaCO}_{2}$ abaixo de $30 \mathrm{~mm} \mathrm{Hg}$, 2- Manitol a $20 \%$ endovenoso, em bolo e infusão rápida, na dose de 1 g/kg, 3- Aumento progressivo da sedação.

A partir de 1992, o tratamento específico para a HIC foi otimizado através da medida intermitente da extração cerebral de $\mathrm{O}_{2}$ (diferença entre a saturação da oxihemoglobina arterial e do sangue venoso jugular, colhido por meio de cateter posicionado no bulbo da veia jugular interna) $)^{6-8}$.

Todos os pacientes foram submetidos a TC de controle após 48 horas do procedimento de monitorização da PIC ou imediatamente após eventual piora clínica. Foi realizada punção cervical lateral C1-C2 para coleta de líquido cefalorraquidiano (LCR), nos pacientes que apresentaram sinais clínicos de suspeita de infecção do sistema nervoso central (SNC), como febre de origem indeterminada, tempo de monitorização acima de 3 dias ou piora clínica, cujos níveis de PIC não contra-indicassem o procedimento (a punção foi realizada somente em pacientes com níveis abaixo de $20 \mathrm{~mm} \mathrm{Hg}$ ).

Nos pacientes que apresentaram sinais clínicos de infecção, nos quais não foi possível a coleta de LCR pelos níveis elevados da PIC, foram introduzidos antibióticos com espectro e penetração adequados para possíveis infecções do SNC, sendo a continuidade da monitorização cuidado- samente avaliada, uma vez que estes pacientes com HIC seriam os que, pelo menos teoricamente, mais se beneficiariam com a monitorização da PIC.

O parafuso metálico foi retirado com técnica asséptica, abertura dos pontos do couro cabeludo, desrosqueamento do parafuso do osso com chave apropriada e ressutura do couro cabeludo. $O$ cateter foi retirado através da contra-abertura, após a liberação do ponto de fixação ao couro cabeludo, necessitando apenas de cuidados habituais de antissepsia.

Foi realizada análise comparativa entre grupos de pacientes divididos de acordo com os níveis da PIC e o resultado final de alta através do teste do qui-quadrado. Valor probabilístico $(p)$ menor que 0,05 foi considerado estatisticamente significativo.

\section{RESULTADOS}

Não foram observadas complicações hemorrágicas adicionais ou hematomas intracranianos relacionados ao método de monitorização da PIC. Em 2 pacientes houve introdução acidental do cateter no parênquima cerebral, tendo ambos apresentado inchaço cerebral intenso no momento da passagem do cateter. Nesses pacientes, o cateter foi imediatamente reimplantado após a observação do seu posicionamento errôneo.

Para controle de possíveis complicações infecciosas relacionadas ao método de monitorização da PIC, foram colhidas amostras de LCR em 66 pacientes, sendo positivas para Acinetobacter sp em 2 pacientes; as restantes foram negativas. Um paciente apresentava evidência, de fístula liquórica nasal e culturas positivas para Acinetobacter calcoaceticus no LCR e nas secreções de orofaringe. $O$ outro paciente, também com evidências de fístula liquórica pelo ouvido e submetido à evacuação cirúrgica de hematoma subdural agudo no $1^{\circ}$ dia pós-TCE, apresentou cultura positiva para Acinetobacter baumanii no LCR.

O resultado final, na alta hospitalar, mostrou 75 óbitos $(36,40 \%)$ e $131(63,60 \%)$ sobreviventes sendo. Destes, $38(18,45 \%)$ apresentavam 8 pontos ou menos na ECGI, $45(21,85 \%)$ de 9 a 12 pontos e 48 $(23,30 \%)$ de 13 a 15 pontos.

A Tabela 1 mostra a influência dos níveis da PIC sobre resultado final do tratamento do TCE grave.

\section{DISCUSSÃO}

No HC-UNICAMP, hospital público estadual, os custos são fatores limitantes importantes para aquisição e manutenção de equipamentos. Em 1989, nossa UTI já contava com equipamentos e pessoal treinado, com experiência de 5 anos, para a monitorização hemodinânica com cateter de Swan-Ganz. 
Tabela 1. Influência dos níveis de PIC sobre o resultado (ECGI na alta hospitalar).

\begin{tabular}{lccccc}
\hline Níveis de PIC & Óbito & $3-8$ & $9-12$ & $13-15$ & Total \\
\hline$\leq 20 \mathrm{~mm} \mathrm{Hg}$ & 4 & 13 & 22 & 30 & 69 \\
$20-40 \mathrm{~mm} \mathrm{Hg}$ & 15 & 16 & 16 & 16 & 63 \\
$>40 \mathrm{~mm} \mathrm{Hg}$ & 56 & 9 & 7 & 2 & 74 \\
Total & 75 & 38 & 45 & 48 & 206 \\
\hline
\end{tabular}

qui-quadrado $=89,479$ (6 graus de liberdade) $p<0,001$.

Esses fatores foram decisivos para a adaptação da monitorização da PIC aos recursos técnicos e humanos já existentes na UTI. A racionalização do uso de salas do centro cirúrgico levou-nos à implantação dos captores de PIC à beira do leito, com material exclusivo para esse fim e com os rigores da técnica asséptica utilizada na passagem do cateter de SwanGanz ${ }^{9}$. A implantação de cateteres subaracnóideos à beira do leito não constituiu fator de risco adicional para complicações infecciosas, como já observado por Yano e col ${ }^{10}$.

O parafuso metálico de aço inoxidável foi utilizado nos primeiros 11 pacientes e mostrou alguns inconvenientes como uma tendência de "achatamento" da curva de PIC, principalmente em níveis mais elevados, com necessidade de algumas irrigações do sistema, com aumento dos riscos de contaminação do sistema e descompensão da $\mathrm{PIC}^{11-13}$. Inconvenientes adicionais observados com o uso do parafuso foram o seu alto perfil, que dificultou a mobilização dos pacientes, além do aparecimento de artefatos metálicos indesejáveis na TC de controle ${ }^{14,15}$. Parafusos plásticos e de baixo perfil foram desenvolvidos para minimizar esses inconvenientes ${ }^{14,16}$. Outros riscos adicionais foram observados no procedimento de retirada do parafuso, realizada necessariamente com técnica asséptica, com a reabertura dos pontos e nova exposição da dura-máter e tecido cerebral ao meio ambiente ${ }^{17}$.

O cateter plástico, confeccionado de cloreto de polivinil (PVC), é de custo desprezível, inextensível o suficiente para não interferir na captação e transmissão da pressão, mas adequadamente flexível para o seu correto posicionamento e fixação ${ }^{18}$. Mostrouse de fácil implantação, inclusive à beira do leito, e com boa biocompatibilidade. O PVC apresenta como características principais ser repelente à água e não aderir aos tecidos, propiciando uma manipulação fácil e indolor. Pode ser utilizado por muitos dias sem risco de trauma ou infecção, levando, graças à sua flexibilidade, a um posicionamento de baixo perfil, o que facilita a mobilização do paciente ${ }^{19}$. Nossas observações estão de acordo com essas características, não tendo sido observadas aderências, reações inflamatórias ou infecciosas teciduais no couro cabeludo relacionadas ao cateter no momento de sua retirada pela contra-abertura, em 195 pacientes monitorizados por até 14 dias. A possibilidade de tunelamento subcutâneo do cateter tem sido considerado importante fator protetor contra contaminação bacteriana e infecções, tanto durante o seu uso quanto no momento da sua retirada ${ }^{20,21}$.

O cateter plástico, além de uma monitorização mais estável raramente necessitando de irrigações, da ausência de artefatos à TC de controle e da maior facilidade para a mobilização dos pacientes, permitiu um procedimento de retirada muito mais prático, rápido e seguro que o parafuso metálico. Por esses motivos, o cateter plástico tem sido a metodologia de primeira escolha para a monitorização da PIC em nossa UTI até hoje ${ }^{8}$.

O cateter ventricular foi o procedimento pionei$\mathrm{ro}^{22}$ e é considerado tradicionalmente como o "padrão-ouro" de fidedignidade da monitorização da PIC, servindo como modelo de comparação para todos os outros métodos propostos, sendo também o único que possibilita a drenagem de LCR, tanto para a análise bioquímica, citológica e microbiológica, quanto como medida para controle da HIC $^{15,16,23}$. As dificuldades técnicas para a punção dos ventrículos, principalmente aqueles comprimidos, deslocados e distorcidos presentes em muitos pacientes com TCE, além dos riscos de lesões adicionais do parênquima e ventriculites, levaram à proposição de outros métodos de monitorização ${ }^{15,17,24}$. A principal vantagem do cateter ventricular, que é a possibilidade da drenagem terapêutica de LCR na HIC, foi questionada por alguns autores, tendo sido levantada a possibilidade de ocorrerem medidas da PIC falsamente menores após essas drenagens ${ }^{25}$.

O método epidural, apesar da facilidade na implantacão e de apresentar os menores riscos de in- 
fecções e pelo lesões de parênquima, é o método de menor fidedignidade, pela interposição da duramáter e pelo riscos do fenômeno de "cunha", que é a pressão adicional sobre o captor, secundária ao descolamento inadequado da meninge ou malposicionamento do captor, com desnivelamento entre este e a dura-máter. Os custos na aquisição e manutenção do equipamento são também consideráveis $^{15,17,24}$.

Os monitores de fibra óptica tiveram o seu uso bastante difundido a partir do final da década de 80. Apesar da vantagem da possibilidade de posicionamento do captor no ventrículo, espaço subaracnóideo ou parênquima, e ausência dos artefatos relacionados com a transmissão por coluna líquida, estes monitores não podem ser recalibrados in situ, acumulando erros de leitura com o passar do tempo, devendo ser reimplantados após cinco dias de uso ${ }^{15,17,24,26}$. Seu custo pode representar também fator limitante para sua aquisição e manutenção.

Apesar de algumas restrições sobre a precisão dos dados do parafuso ${ }^{11}$ e do cateter ${ }^{27}$, o método subaracnóideo figura como um método de fidedignidade bastante satisfatória, relativa facilidade de implantação e mínimos riscos de lesões adicionais e infecções, além de apresentar custos de aquisição e manutenção bastante reduzidos ${ }^{9,15,17,24}$. A precisão do método subaracnóideo, principalmente com cateteres, foi confirmada por Yano e col. ${ }^{10}$, Mollman e col. ${ }^{13}$, Wilkinson ${ }^{20}$, Villanueva ${ }^{28}$ e Sugiura e col $^{29}$.

A principal desvantagem relacionada ao método, nesta casuística, foi a grande dificuldade para a coleta de LCR, tanto pelo parafuso quanto pelo cateter, o que impossibilitou a análise bioquímica, citológica e microbiológica desse material. Mesmo quando foi possível essa coleta em raros casos, as amostras não foram adequadas para a análise, uma vez que evidenciaram graus variados de degeneração citológica e coágulos, secundários à estase no interior do sistema. Por outro lado, este tipo de procedimento tem sido desaconselhado uma vez que a aspiração de LCR pelo cateter aumenta o risco de colonização do sistema e infecção ${ }^{10}$. As culturas da ponta dos cateteres também foram inadequadas por evidenciarem invariavelmente contaminação por bactérias da pele, apesar de todos os cuidados na sua retirada ${ }^{10}$.

O controle liquórico foi realizado em 66 pacientes segundo critérios clínicos quando os níveis de PIC não contra-indicaram a punção. A técnica de punção cervical lateral $\mathrm{C} 1-\mathrm{C} 2^{30}$ foi utilizada também pelas dificuldades importantes, proibitivas até em alguns casos, para o posicionamento adequado dos pacientes para as punções convencionais suboccipital e lombar. Essas dificuldades incluem a presença do tubo traqueal, múltiplos acessos arteriais e venosos, trações, drenos e monitorizações variadas, além das possíveis repercussões indesejáveis do posicionamento requerido para as punções convencionais sobre a PIC.

$O$ diagnóstico de meningite em pacientes neurocirúrgicos é freqüentemente difícil, uma vez que apresentam, em sua maioria, reação inflamatória inespecífica no LCR (com pleocitose e aumento de proteínas) ${ }^{31}$. Desse modo, o diagnóstico definitivo de meningite nestes pacientes foi estabelecido pela presença de micoorganismos no $\mathrm{LCR}^{31-34}$. As infecções encontradas em dois pacientes desta casuística não puderam ser relacionadas exclusivamente ao método de monitorização da PIC, uma vez que apresentavam outros fatores de risco de infecção do SNC, como fístula liquórica e neurocirurgia prévia ${ }^{32-34}$.

Em dois casos foi identificada introdução acidental do cateter no parênquima cerebral, não sendo evidenciadas outras complicações relacionadas ao método de monitorização da PIC. Frente a esses dados, o método subaracnóideo foi considerado seguro e com riscos mínimos, o que está de acordo com o observado por Yano e col. ${ }^{10}$, Wilkinson ${ }^{20}$, Vilanueva ${ }^{28}$, Sugiura e col. ${ }^{29}$ e Narayan e col. ${ }^{32}$.

A anotação do valor da PIC de final de hora em ficha padronizada pelo pessoal de enfermagem, apesar da menor sensibilidade, tem sido considerado um método satisfatório para a estimativa da evolução da PIC da "hora inteira", além de ser uma alternativa mais prática e econômica de registro desses valores. Com variações insignificantes dos valores continuamente registrados por métodos computadorizados complexos ${ }^{35}$, essa metodologia foi também padronizada pelos pesquisadores do Traumatic Coma Data Bank ${ }^{23}$.

Dois terços dos pacientes estudados $(66,50 \%)$ apresentaram níveis de PIC acima de $20 \mathrm{~mm} \mathrm{Hg}$; metade $(51,82 \%)$ desses pacientes evolui para óbito, o que demonstra a alta incidência de HIC em pacientes com TCE e sua influência deletéria na evolução desses pacientes ${ }^{23}$.

A significativa influência dos níveis de PIC sobre o resultado final desta casuística $(p<0,001)$, confirma a importância da identificação da HIC o mais precocemente possível. Todos os esforços devem ser voltados para o seu controle, tanto através de medidas cirúrgicas quanto através de medidas medicamentosas ${ }^{23,32}$. 


\section{CONCLUSÕES}

Baseados na análise desta casuística, concluímos que o método subaracnóideo para a monitorização contínua da PIC mostrou-se aplicável, sendo considerado seguro, simples e de baixo custo. $O$ registro dos níveis de PIC de horário pelo pessoal de enfermagem mostrou-se uma metodologia prática e útil para a condução do tratamento dos pacientes. A HIC mostrou-se uma complicação freqüente em pacientes com TCE grave, apresentando uma forte influência negativa sobre os resultados do tratamentos desses pacientes.

Observamos que o cateter plástico apresentou vantagens de ordem prática sobre o parafuso metálico no que diz respeito a estabilidade das monitorizações, manipulação do paciente, procedimento de retirada e produção de artefatos na TC, apesar do pequeno número de pacientes monitorizados com parafuso.

A padronização de um método de monitorização contínua da PIC deve ser uma preocupação premente em qualquer UTI, principalmente em hospitais públicos, responsáveis pelo tratamento da maioria dos pacientes com TCE em nosso país. O método subaracnóideo, especialmente com cateter plástico, além de satisfazer as necessidades específicas de segurança, praticabilidade e confiabilidade, contempla também os grandes desafios da falta de recursos financeiros na área de assistência à saúde em nosso meio. Este método deve, portanto, ser sempre considerado como uma alternativa viável para a monitorização da PIC em unidades de terapia intensiva.

\section{REFERÊNCIAS}

1. TeasdaleG, Jennet B. Assessment of coma and impaired consciousness: a practical scale. Lancet 1974;2:81-84.

2. Dantas VP Filho. Aspectos técnicos da monitorização da pressão intracraniana pelo método subaracnóideo e análise dos fatores que influenciaram a evolução de 206 pacientes com traumatismo craniencefálico grave. Tese Unicamp. Campinas:1999.

3. Dantas VP Filho, Fal cão ALE. Monitorização da pressão intacraniana. In Terzi RGG, A raújo S (eds). Monitorização invasiva em UTI. Campinas: Scan, 1991:131-141.

4. Falcão ALE, Dantas VP Filho, Saraiva JFK, Sardinha LAC, Araújo S, Terzi RGG. Bedside ICP monitoring in head trauma patients by using a simple, safeand low cost methodology. Clin Intens Care 1992;3 Suppl 112 .

5. Andrade AF, Teixeira MJ, Bacheschi LA, et al. Medida contínua da pressão intracraniana com parafusos: modificação técnica. Arq Bras Neurocirurg 1983;2:143-148.

6. Cruz J. Relevância da otimização ventilatória em hipertensão intracraniana aguda: uma abordagem clínica-fisiológica-terapêutica. Arq Neuropsiquiatr 1995;53:131-140.

7. Falcão ALE, Dantas VP Filho, Sardinha LAC, et al. Highlighting intracranial pressure monitoring in patients with severe acute brain trauma. Arq Neuropsiquiatr 1995;53:390-394.

8. Falcão $A L E$, Araújo $S$, Dragosavac $D$, et al. Hemometabolismo cerebral: variações na fase aguda do coma traumático. Arq N europsiquiatr 2000;58:877-882.
9. Flitter MA. Techniques of intracranial pressure monitoring. Clin Neurosurg 1980:28:547-563.

10. Yano M, Kobayashi S, Otsuka, T. Useful ICP monitoring with subarachnoid catheter method in severehead injuries. J Trauma 1988;28:476480.

11. Mendelow AD, Rowan JO, Murray L, Kerr AE. A clinical comparison of subdural screw pressure measurements with ventricular pressure.J Neurosurg 1983;58:45-50.

12. Dearden NM, M CDowall DG, Gibson RM. Assessment of Leeds device for monitoring intracranial pressure. J Neurosurg 1984;60:123-129.

13. Mollman HD, Rockswold GL, Ford SE. A clinical comparison of subarachnoid catheters to ventriculostomy and subarachnoid bolts: a prospective study. J Neurosurg 1988;68:737-741.

14. Landy HJ, Villanueva PA. An improved subarachnoid screw for intracranial pressure monitoring: technical note. J Neurosurg 1984;61:606-608.

15. Feldman Z, Narayan RK. Intracranial pressure monitoring: techniques and pitfalls.In Cooper PR (ed). Head injury 3. Baltimore: Williams \& Wilkins, 1993:247-274.

16. Becker DP, GadeGF, Young HF, Feuerman TF. Diagnosis and treatment of head injury in adults. In Youmans JR (ed). Neurological surgery 3. Philadel phia: Saunders, 1990:2017-2148.

17. Wilkinson HA. Intracranial pressure. In YoumansJR (ed). Neurological surgery 3. Philadel phia: Saunders, 1990:661-695.

18. Arst DB, Silver $M$, Lahey WJ. Theuse of vinyl plastictubing in recording pressure phenomena. Am Heart J 1951;42:746-748.

19. Rushmer RF. Biomechanics and biomaterials. In Rushmer RF (ed).Medical engineering: projections for health and care delivery. N ew York: A cademic Press, 1972:293-320.

20. Wilkinson HA. The intracranial pressure-monitoring cup catheter: technical note. Neurosurgery 1977;1:139-141.

21. Mendes $M$, Moore $P$, Wheeler CB, Winn HR, Rodeheaver G. Susceptibility of brain and skin to bacterial challenge. J Neurosurg 1980;52:772-775.

22. Lundberg N. Continuous recording and control of ventricular fluid pressure in neurosurgical practice. Acta Psychiat Neurol Scand 1960;36 (Suppl 149).

23. Marmarou A, Anderson RL, Ward JD, et al. Impact of ICP instability and hypotension on outcome in patients with severe head trauma. J Neurosurg 1991;75(Suppl):S59-S66.

24. Luerssen TG, Marshall LF. Themedical management of head injury. In Vinken PJ, Bruyn GW, Klawans HL, Braakman R (eds). Handbook of clinical neurology: head injury. A msterdame: Elsevier, 1990;57(revised series 13):207-247.

25. Wilkinson HA, Yarzebski J, Wilkinson EC, Anderson FA. Erroneous measurement of intracranial pressure caused by simultaneous ventricular drainage: a hydrodynamic model study. Neurosurgery 1989;24:348-354.

26. Crutchfield JS, Narayan RK, Robertson CS, Michael LH. Evaluation of a fiberoptic pressure monitor. J Neurosurg 1990;72:482-487.

27. Barlow $P$, Mendelow $A D$, Lawrence AE, Barlow $M$, Rowan JO. Clinical evaluation of two methods of subdural pressure monitoring. J Neurosurg 1985;63:578-582.

28. VillanuevaPA. Simplified techniquefor subdural pressuremonitoring: technical note. Neurosurgery 1985;16:238-240.

29. Sugiura K, Hayama N, Tachisawa T, Baba M, Takisawa H. Intracranial pressure monitoring by a subdurally placed silicone catheter: technical note. Neurosurgery 1985;16:241-244.

30. Zivin JA. Lateral cervical puncture: an alternative to lumbar puncture. Neurology 1978;28:616-618.

31. StephensJL, PeacockJE. Uncommon infections: eyeand central nervous system. In Wenzel RP (ed). Prevention and control of nosocomial infections 2. Baltimore: Williams \& Wilkins, 1993:746-775.

32. Narayan RK, Kishore PRS, Becker DP, et al. Intracranial pressure: to monitor or not to monitor? A review of our experience with severe head injury. J Neurosurg 1982;56:650-659,.

33. Mayhall CG, Archer NH, Lamb A, et al. Ventriculostomy-related infections: a prospective epidemiologic study. N Engl J Med 1984;310:553-559.

34. Aucoin PJ, Kotilainen HR, Gantz N M, Davidson R, Kellogg P, Stone B. Intracranial pressure monitors: epidemiologic study of risk factors and infections. Am J Med 1986;80:369-376.

35. Turner HB, Anderson RL, Ward JD, Young HF, Marmarou A. Comparison of nurse and computer recording of ICP in head injured patients. J Neurosci Nurs 1988;20:236-239. 